\title{
Lighter or Heavier Than Predicted: Neural Correlates of Corrective Mechanisms during Erroneously Programmed Lifts
}

\author{
Per Jenmalm, ${ }^{1,2,5}$ Christina Schmitz, ${ }^{1,4}$ Hans Forssberg, ${ }^{1}$ and H. Henrik Ehrsson ${ }^{1,3}$ \\ ${ }^{1}$ Neuropediatric Unit, Department of Women and Child Health, Karolinska Institutet, SE-17177 Stockholm, Sweden, ${ }^{2}$ Department of Hand and Plastic \\ Surgery, Norrlands University Hospital, SE-90185 Umeå, Sweden, ${ }^{3}$ Wellcome Department of Imaging Neuroscience, Institute of Neurology, London WC1N \\ 3BG, United Kingdom, ${ }^{4}$ Centre National de la Recherche Scientifique-Groupe Développement et Pathologie de l'Action, 13402 Marseilles, France, and \\ ${ }^{5}$ Department of Integrative Medical Biology, Umeå University, SE-90187 Umeå, Sweden
}

A central concept in neuroscience is that the CNS signals the sensory discrepancy between the predicted and actual sensory consequences of action. It has been proposed that the cerebellum and parietal cortex are involved in this process. A discrepancy will trigger preprogrammed corrective responses and update the engaged sensorimotor memories. Here we use functional magnetic resonance imaging with an event-related design to investigate the neuronal correlates of such discrepancies. Healthy adults repeatedly lifted an object between their right index fingers and thumbs, and on some lifting trials, the weight of the object was unpredictably changed between light $(230 \mathrm{~g})$ and heavy $(830 \mathrm{~g})$. Regardless of whether the weight was heavier or lighter than predicted, activity was found in the right inferior parietal cortex (supramarginal gyrus). This suggests that this region is involved in the comparison of the predicted and actual sensory input and the updating of the sensorimotor memories. When the object was lighter or heavier than predicted, two different types of preprogrammed force corrections occurred. There was a slow force increase when the weight of the object was heavier than predicted. This corrective response was associated with activity in the left primary motor and somatosensory cortices. The fast termination of the excessive force when the object was lighter than predicted activated the right cerebellum. These findings show how the parietal cortex, cerebellum, and motor cortex are involved in the signaling of the discrepancy between predicated and actual sensory feedback and the associated corrective mechanisms.

Key words: precision grip; cerebellum; parietal cortex; dexterous manipulation; fingertip force control; motor cortex

\section{Introduction}

The human ability to manipulate objects relies on predictive control mechanisms that parametrically adapt the force motor commands to the relevant physical properties of the target object (Johansson, 1998). These physical properties are stored as sensorimotor memories and are acquired during previous manipulatory experience (Johansson and Cole, 1992). However, in everyday life, object properties may be unpredictable because they can change without our knowledge. When the weight of a lifted object is unpredictably changed, the applied finger tip forces will either be too large or too small (Johansson and Westling, 1988), and the afferent sensory information will deviate from the centrally predicted outcome. Detection of such a mismatch triggers fastpreprogrammed patterns of corrective responses along with an

Received Nov. 28, 2005; revised May 22, 2006; accepted May 31, 2006.

This work was supported by the Swedish Research Council (Grants 5925 and 8667). C.S. was awarded a MarieCurie research grant by the European Union. H.H.E. was supported by the Human Frontier Science Program. We thank Anders Bäckström for technical support and Roland Johansson for discussions and comments on this manuscript.

Correspondence should be addressed to H. Henrik Ehrsson, Wellcome Department of Imaging Neuroscience, Institute of Neurology, 12 Queen Square, London WC1N 3BG, UK. E-mail: h.ehrsson@fil.ion.ucl.ac.uk. D01:10.1523/JNEUROSCI.5045-05.2006

Copyright $\odot 2006$ Society for Neuroscience $\quad$ 0270-6474/06/269015-07\$15.00/0 update of the relevant sensorimotor memories used for the predictive control (Johansson and Westling, 1988).

The present study was designed to reveal central neural activities associated with unpredictable weight changes in a lifting task and the neural circuits involved in the fast corrective responses triggered in lifts after the unpredicted weight changes. We registered brain activity in healthy subjects associated with individual lifts using functional magnetic resonance imaging (fMRI). By comparing the brain activity from trials performed with a heavy weight, but programmed for a lighter weight, with trials that were adequately programmed for the heavy weight, we could elucidate the neural substrates responsible for the slow, discontinuous increases in fingertip force that occur after the initial program for the light weight has failed to lift the object (Johansson and Westling, 1988; Gordon et al., 1991, 1993). Likewise, by comparing the brain activity in trials performed with a light weight, but programmed for a heavier weight, with trials that were adequately programmed for a light weight, we could elucidate the neural substrates responsible for the abrupt termination of the fingertip force that occurs after the premature lift off (Johansson and Westling, 1988). We hypothesized that the primary sensorimotor cortex and the cerebellum would be involved in these corrective mechanisms because they receive strong somatosen- 
sory input from the fingertips and control hand muscle activity (Picard and Smith, 1992; Maier et al., 1993; Dettmers et al., 1995; Lemon et al., 1998; Monzee and Smith, 2004)

Brain areas that were activated during the unpredictable weight changes, regardless of whether the weight is heavier or lighter, would likely represent neural circuits involved in the mismatch detection between the predicted and actual sensory information and the subsequent updating of the sensorimotor memories (Johansson, 1998; Wolpert and Ghahramani, 2000). Based on previous studies, we hypothesized that the cerebellum (Doya, 1999; Kawato, 1999; Blakemore et al., 2001), and frontal and parietal areas associated with object manipulation (Schmitz et al., 2005), would show such increases in synaptic activity.

\section{Materials and Methods}

\section{Subjects and experimental procedure}

Experiments were conducted on 12 right-handed subjects (mean age, $29 \pm 3$ years; 7 males and 5 females). All subjects had given their written informed consent, and the study was approved by the Ethical Committee of the Karolinska Hospital. The nonmagnetic-instrumented test object has been described previously (Schmitz et al., 2005). Briefly, it had two vertical flat parallel contact surfaces that were covered with sandpaper. The experimenter, adding or removing an extra load of $600 \mathrm{~g}$ outside of the MR scanner, could easily change the weight of the object. The total weight of the test object could have one of two values, $230 \mathrm{~g}$ (light) or $830 \mathrm{~g}$ (heavy). The grip force normal to the contact surfaces and the vertical lift force tangential to the grip surface, together with the vertical position of the object, were registered continuously by a fiber optics system.

While lying in the MR scanner, the subjects used the tips of their right index fingers and thumbs in a precision grip to lift the test object a few centimeters above a support and then replace it. The subjects lifted the object 58 times in one run, and each lift was paced by a sound that lasted for $2 \mathrm{~s}$. There was a $5 \mathrm{~s}$ pause between each lift when the subjects relaxed their hands. The weight of the object was unpredictably changed 16 times in each series (i.e., after two, three, or four lifts with the same weight). Before scanning, subjects were trained to correctly lift the object. The order of weight changes was varied across subjects and series.

\section{Behavioral analysis}

Using a flexible data acquisition and analysis system (SC/ZOOM; Section of Physiology, IMB, Umeå University), the force and position signals (400 samples/s) were sampled. The timing information about the acquisition of each MR image was recorded and stored together with the force and position data, thereby eliminating any timing error when later comparing the behavioral and the imaging data. The reported grip and vertical lift forces refer to the mean value of the corresponding forces at the two grip surfaces. The moment of contact and object liftoff was determined by an off-line inspection of the grip force and vertical position trace.

The load phase was defined for each lifting trial and was calculated as the time period between touch and liftoff. Static grip and lift forces were calculated as the mean forces during the time interval between 0.8 and $1 \mathrm{~s}$ after touch when the object was held in the air. The end of this phase was determined by visual inspection and later used as events for brain imaging analysis. The peak rate of grip force increase during the load phase was assessed from the first time derivative of the grip force signal using a \pm 5 point numerical differentiation (i.e., force rate was calculated within a window of $\pm 12.5 \mathrm{~ms}$ ). Repeated-measures ANOVAs were used to evaluate the influence of current object weight ( 230 or $830 \mathrm{~g}$ ) and the weight of the previous lift ( 230 or $830 \mathrm{~g}$ ) on the load phase, static grip force, and the maximum grip force rate during the load phase. The level of probability selected as statistically significant was $p<0.05$. Values reported in the figures for data pooled across trials for all subjects refer to means \pm SD.

\section{Brain imaging}

fMRI was performed on a $1.5 \mathrm{~T}$ scanner (Sigma Horizon Echospeed; General Electric Medical Systems, Milwaukee, WI) equipped with a head coil. A plastic bite bar was used to restrict head movements. A highresolution T1-weighted anatomical image volume of the entire brain (3D-SPGR) and functional gradient-echo, echo-planar $\mathrm{T} 2{ }^{\star}$-weighted images with blood oxygenation level-dependent (BOLD) contrast (field of view, $22 \mathrm{~cm}$; matrix size, $64 \times 64$; pixel size, $3.4 \times 3.4 \mathrm{~mm}$; echo time, $50 \mathrm{~ms}$; flip angle, $90^{\circ}$ ) were collected. A functional image volume comprised 30 slices of $5.4 \mathrm{~mm}$ thickness, which covered the entire brain. Four experimental runs were conducted for each subject. Each run, lasting $408 \mathrm{~s}$ [repetition time (TR), $4 \mathrm{~s}$ ], included a total of 102 functional volumes. Functional image volumes were collected in four separate runs. In each run lasting $408 \mathrm{~s}, 110$ volumes were acquired continuously, with one volume being collected every $4000 \mathrm{~ms}$ (TR, $4 \mathrm{~s}$ ). By jittering the lift events and the acquisition of the MRI scans, an effective temporal resolution of $\sim 1 \mathrm{~s}$ was achieved. To allow for $\mathrm{T} 1$ equilibration effects, six volumes were recorded before each run and were discarded.

Images were processed and analyzed using the Statistical Parametric Mapping software (SPM99; http://www.fil.ion.ucl.ac.uk/spm/; Wellcome Department of Cognitive Neurology, London, UK). The functional images were realigned to correct for head movements, coregistered to each individual anatomical T1-weighted image, and normalized (transformed by nonlinear transformations) to the standard space of the Montreal Neurological Institute. The functional images were spatially smoothed with an $8 \mathrm{~mm}$ full-width at half-maximum isotropic Gaussian filter.

Individual subject analysis (level 1). We applied a general linear model using regressors for these events: (1) "Change to lighter," when the object's weight was changed to light after a series of at least two consecutive lifts of a heavy object; (2) "Change to heavier," when the object's weight was changed to heavy after a series of at least two consecutive lifts of a light object; (3) "No change light," when the object's weight was light after a series of at least two consecutive lifts of a light object; and (4) "No change heavy," when the object's weight was heavy after a series of at least two consecutive lifts of a heavy object. The other lifts were modeled as events of no interest. All covariates were convolved by the standard SPM canonical hemodynamic response function. The movement parameters estimated during the realignment preprocessing were included in the model to eliminate movement-related artifacts that could not be corrected by rigid-body realignment.

Parameter estimates and variances were derived for each covariate in a subject-specific fixed-effects model. Our experimental design used a $2 \times$ 2 factorial design with the two factors being change of object (change or no change) and object weight (heavy or light). Thus, we examined the main effect of changing the object and the interaction between change of object and object weight. To locate areas related to the change in object, irrespectively of whether the object was lighter or heavier than predicated, we examined the main effect of changing an object using the following contrast: [(Change to lighter - No change light $)+($ Change to heavier - No change heavy)]. We also looked for areas showing differential responses when the weight of the object changed to lighter or heavier using these interaction contrasts: [(Change to lighter - No change light $)-($ Change to heavier - No change heavy)] and [(Change to heavier - No change heavy) - (Change to lighter - No change light)], respectively.

Random-effect analysis (level 2). To accommodate for intersubject variability in group analysis, the contrast images from level 1 were entered into a second-level $t$ test, to create an SPM $\{\mathrm{t}\}$. A one-sample $t$ test was used (11 degrees of freedom). To generate the statistical parametric maps, we used a threshold of $p<0.001$ at each voxel. For the statistical inference, we report clusters of voxels that corresponded to $p<0.05$ after correction for multiple comparisons in the entire brain (Family-WiseError correction). For the left primary sensorimotor cortex $(-36,-24$, 51 ), bilateral cerebellum, and right posterior parietal cortex, where we had strong a priori hypotheses, we used small volume corrections using peaks taken from a previous study: left primary motor cortex: sphere at $-36,-24,51$ (20 $\mathrm{mm}$ radius); bilateral cerebellum: sphere at $\pm 33,-63$, -30 (20 mm radius); right inferior parietal cortex: sphere at 57, $-33,30$ (20 mm radius) (Schmitz et al., 2005). 

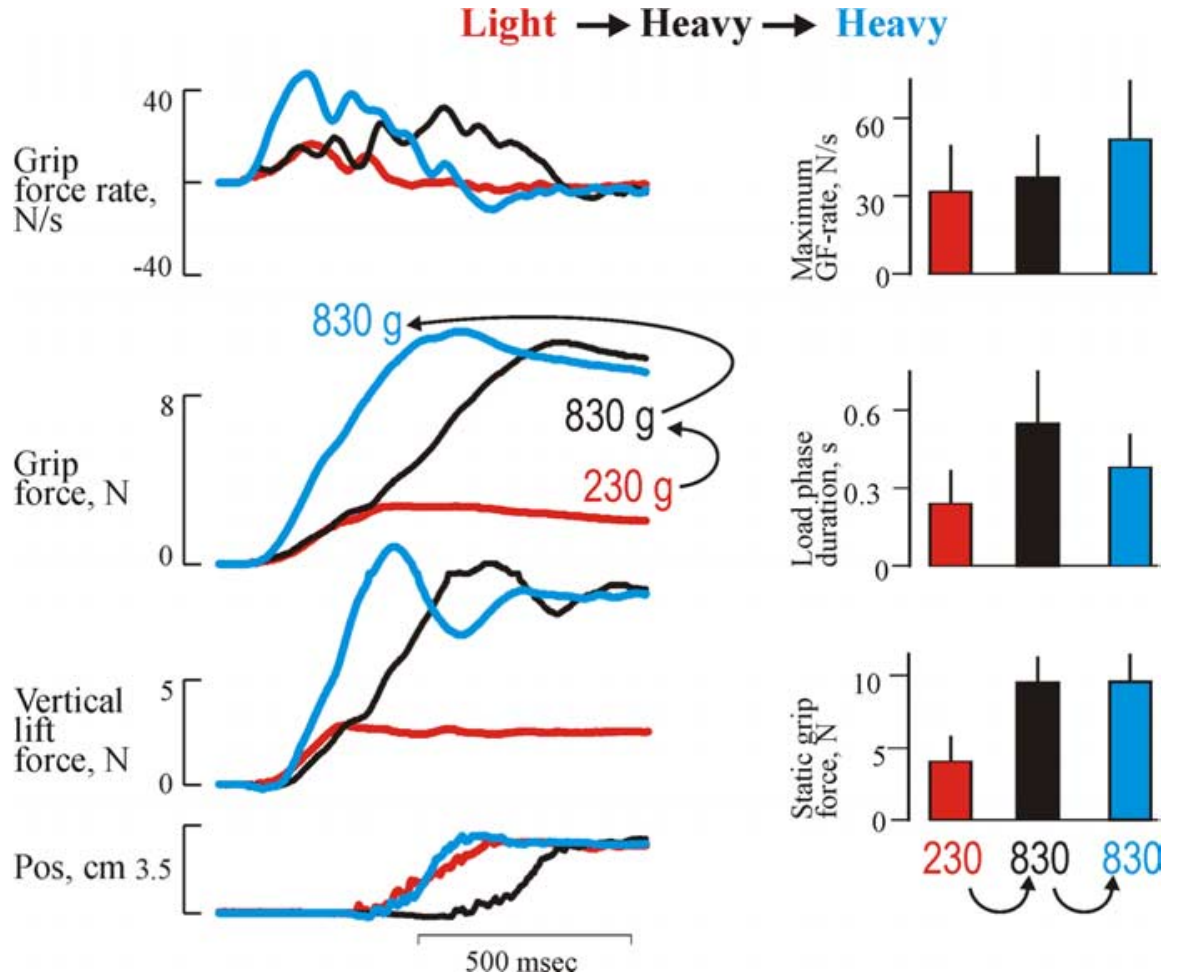

Figure 1. Behavioral recordings from erroneously programmed lifts with the heavy weight versus adequately programmed lifts (Change to heavier - No change heavy). The grip force (GF), vertical lift force, and grip force rate and vertical position as a function of time for the adjustment to a heavy weight in three single trials are shown. The histograms in the right panels represent mean static grip forces, maximum grip force rates, load phase duration for the heavy weight, and the influences of weight of the preceding trial: heavy (blue) and light (black). For comparison, the light weight preceded with a light weight is also illustrated (orange). The vertical bars at the top of each column gives +1 SD. Data are pooled from all subjects.

\section{Results}

\section{Behavioral performance}

The performance of the precision grip lifts in the fMRI scanner corresponded well with the original study by Johansson and Westling (1988). When an unpredictable heavy weight was lifted after a light weight, the forces followed the trajectories of the preceding light trial until the predicted liftoff. Thereafter, the forces slowly continued to increase until the vertical lift force overcame gravity and the object started to move (Fig. 1). As a consequence, this lift showed a smaller grip force rate, and the object started to move later; therefore, the duration of the loading phase was prolonged compared with heavy weight lifts that were preceded by the same weight $(p<0.05)$ (Fig. 1$)$. When an unpredictable light weight was lifted after a heavy weight, the forces during the load phase followed the trajectories of the preceding heavier weight, resulting in an early lift off (Fig. 2). Because of the reaction time of the triggered correction, this lift showed a pronounced overshoot in the grip force and the vertical position of the object (Fig. 2). Therefore, the load phase duration was shorter and the maximum grip force rate was larger compared with trials performed with the light weight and that were preceded by the same weight $(p<0.05)$. After this, the grip force increase and the upward movement were terminated, and the grip force slowly decayed.

\section{Brain activity}

Main effect of changing object: heavier and lighter than predicted First, we looked for areas that showed increases in activity associated with the unpredictable weight changes, irrespective of whether the object became lighter or heavier than predicted [(Change to lighter - No change light) + (Change to heavier - No change heavy)]. Activation was found in the right supramarginal gyrus of the inferior parietal lobe (Fig. 3, Table 1).

\section{Lighter than predicted}

Next, we looked for areas that were active when the weight of the object was lighter than predicted. These lifts were associated with increased activity in the right cerebellar hemisphere compared with the lifts preceded by the same weight (Change to lighter - No change light; $p<0.05$ corrected) (supplemental Fig. 2 and supplemental Table 2, available at www. jneurosci.org as supplemental material). More importantly, the same section of the right cerebellum was active when we directly contrasted lifting trials when the object was lighter than predicted compared to when it was heavier than predicted ( $p<0.05$ corrected) (Fig. 4, Table 2) [(Change to lighter - No change light $)-$ (Change to heavier - No change heavy)]. Thus, the cerebellar activity was specifically related to the corrective response when the weight was unexpectedly light.

\section{Heavier than predicted}

Finally, we identified active areas associated with lifts when the object was heavier than predicted. The primary motor cortex (M1) and primary somatosensory cortex (S1) was active when the participants lifted the object that was heavier than predicted compared with a heavy weight lift that was preceded by the same weight (Change to heavier - No change heavy; $p<0.05$ corrected) (supplemental Fig. 1 and supplemental Table 1, available at www.jneurosci.org as supplemental material). Moreover, the same areas were active when we directly compared the lifts when the object was heavier than predicted with those when it was lighter than predicated [i.e., M1 and S1; peaks located in the left central sulcus, the left precentral gyrus, and the left postcentral gyrus; (Change to heavier - No change heavy) - (Change to lighter - No change light); $p<0.05$ corrected] (Fig. 4, Table 2). Thus, the activity in M1 and S1 seemed to be specifically related to the force responses when the object was heavier than predicted.

\section{Discussion}

In this study, we can associate activity in the motor cortex, cerebellum, and inferior parietal cortex with different aspects of the sensorimotor control mechanisms of object manipulation. First, the right supramarginal gyrus was active after an unpredicted change of the object weight regardless of whether it was lighter or heavier than predicted. We suggest that this activity reflects the neural monitoring of a mismatch between the centrally predicted and the actual sensory input, and the associated neural processing related to the updating of the sensorimotor memories (see Introduction). Second, when the weight of the object suddenly increased, but not when it decreased, there was increased activity in the left (contralateral) primary motor and somatosensory (S1/ 
M1) cortices. We suggest that these areas are involved in the slow increase of the grip and vertical lift force that occur after the expected liftoff. Finally, the right (ipsilateral) cerebellum seems to be specifically active when the weight of an object is suddenly decreased. This area probably mediates the fast termination of the increase in fingertip forces that occur after the early liftoff.

A potential confounder in the present study relates to automatic changes in attention associated with the unpredictable sensorimotor events. We believe that it is unlikely that changes in attention can explain the activation of the supramarignal gyrus, because this area does not belong to the network of prefrontal, temporal, and other parietal areas that has been associated with attentional responses during unexpected changes in the sensory environment (Downar et al., 2000). In addition, other parts of this attention network were not activated. Rather, this particular part of the inferior parietal cortex has been previously associated with various finger manipulation tasks in which attention has been reasonably matched (Binkofski et al., 1999; Ehrsson et al., 2000, 2001; KuhtzBuschbeck et al., 2001). It should be noted that the "corrective" activations of M1 and cerebellum, respectively, were detected as we made direct comparisons between the lifts when the weight was heavier or lighter than predicted. In both series, the events were equally unexpected, and thus the attentional factor should be the same.

\section{Mismatch between predicted and actual weight and updating of sensorimotor memories}

We found activity in the right supramarginal gyrus associated with weight changes in any direction (lighter or heavier than predicted). This is consistent with our previous fMRI experiment, in which we found right-sided supramarginal activation when the weight of the object was changed in an irregular manner compared with when the same weight was lifted in consecutive lifts (Schmitz et al., 2005). Because a block design was used in the previous study, the present results obtained by an event-related design provide more compelling evidence that the supramarginal cortex is activated at the time point when the weight is actually changed. The updating of the sensorimotor memory representations of objects typically takes place on a single-trial basis during precision grip lifts (Gordon et al., 1993; Jenmalm and Johansson, 1997; Johansson and Westling, 1988). The activity in the supramarginal gyrus may signal the discrepancy between the predicted and actual sensory information (Blakemore and Sirigu, 2003), or the updating of the sensorimotor memories of the object's physical proprieties. Our data are consistent with both of these possibilities, and the supramarginal cortex has the physiological and anatomical properties to implement both monitoring a sensory mismatch and updating sensorimotor memories. It has been found to be activated in several tasks that involve manipulation, lifting, or pinching of objects (Binkofski et al., 1999; Ehrsson et al., 2000, 2001). It is anatomically connected with motor areas
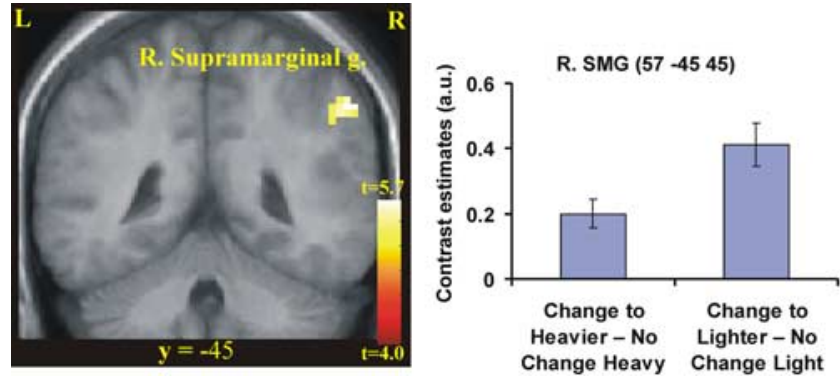

Figure 3. Areas activated when the weight of the object was unpredictably changed, regardless of whether it was lighter or heavier than predicted. We tested the main effect of change in object weight [(Change to heavier - No change heavy) + (Change to lighter - No change light); $p<0.05$ corrected]. Activity was found in the right supramarginal gyrus right (R. Supramarginal g.) of the posterior parietal lobe (right; $p<0.01$ corrected) as shown on a coronal (left). The activations are superimposed on a mean image of the participants' structural scans. For illustrative purposes, we used $p<0.001$ uncorrected and $>5$ voxels to threshold the statistical parametric maps. L, Left hemisphere; $R$, right hemisphere. The coordinate in standard space for the two slices is indicated. In the right panel, we plot the BOLD response in terms of the contrast estimates from the right supramarginal gyrus (R. SMG) for the two contrasts, (Change to heavier - No change heavy) and (Change to lighter - No change light). As can be seen, there is stronger activity during both types of lifts with unpredictable weight changes compared with the well programmed lifts. a.u., Arbitrary units.

including the ventral premotor cortex (Godschalk et al., 1984; Cavada and Goldman-Rakic, 1989; Neal et al., 1990; Ghosh and Gattera, 1995), which is important for the control of precision grips and manipulation (Binkofski et al., 1999; Ehrsson et al., 
Table 1. Main effect of unexpected change in object weight: (Change to heavier No change heavy) and (Change to lighter - No change light)

\begin{tabular}{|c|c|c|c|c|c|c|c|}
\hline \multirow[b]{2}{*}{ Anatomical region } & \multicolumn{3}{|c|}{$\begin{array}{l}\text { Talairach } \\
\text { coordinates }\end{array}$} & \multirow{2}{*}{\multicolumn{2}{|c|}{ Peak Zscore }} & \multirow[b]{2}{*}{ Cluster size } & \multirow[b]{2}{*}{$p$ value $^{a}$} \\
\hline & $x$ & $y$ & $Z$ & & & & \\
\hline Right supramarginal gyrus & 57 & -45 & 45 & $3.88^{b}$ & \} & 23 & 0.05 \\
\hline
\end{tabular}

A

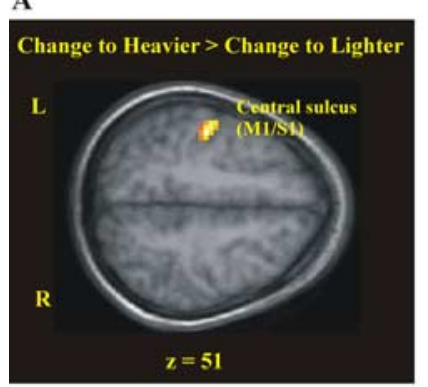

L. $M 1(-45-1851)$

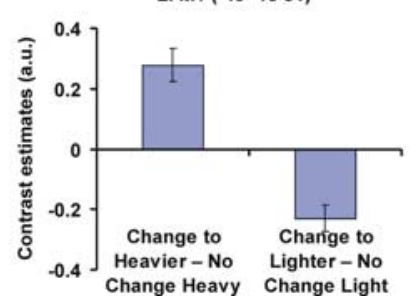

B

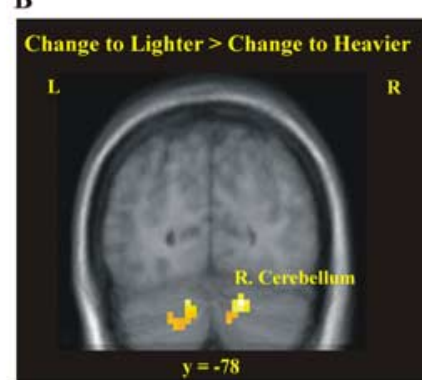

R. cerebellum (15 -78 -27)

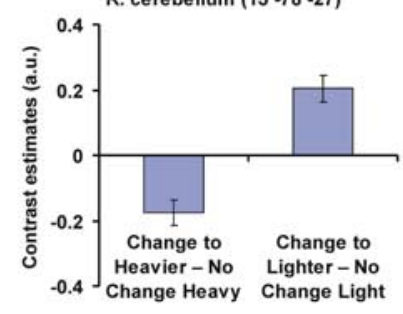

Figure 4. Neural activity in erroneously programmed trials specific for one type of prestructured force response. $\boldsymbol{A}$, The primary motor cortex was more strongly activated on lifts performed with a heavy weight, but programmed for a lighter weight, compared with lifts performed with a light weight, but programmed for a heavier weight [(Change to heavier - No change heavy) - (Change to lighter - No change light); $p<0.05$ corrected]. $\boldsymbol{B}$, The right lateral cerebellum (R. Cerebellum; lobule VI/crus I) showed greater activity on lifts performed with a light weight, but programmed for a heavier weight, than on trials performed with a heavy weight, but programmed for a lighter weight [(Change to lighter - No change light) (Change to heavier - No change heavy); $p<0.05$ corrected]. For illustrative purposes, we used $p<0.001$ uncorrected and $>5$ voxels to threshold the statistical parametric maps. The coordinate in standard space for the two slices is indicated. In the bottom panels, we plot the contrast estimates for the contrasts (Change to heavier - No change heavy) and (Change to lighter - No change light). Note the opposite pattern of responses in M1 and the right supramarginal cortex. L, Left hemisphere; R, right hemisphere; a.u., arbitrary units.

2000). It also receives input from the cerebellum (Clower et al., 2001). Thus, the supramarginal gyrus might be a node that uses information about mismatches between predicated and actual feedback to update the sensorimotor memory representations of manipulated objects that are to be used in the programming of forthcoming manipulative tasks. This view is consistent with our previous findings that the posterior parietal cortex is important for the coordination of fingertip forces during manipulation (Ehrsson et al., 2003). The fact that strong activation is often seen in the right (ipsilateral) inferior parietal cortex (Ehrsson et al., 2000, 2001, 2003), even when people use their right hand in manipulative tasks, may reflect a dominance of the right hemisphere for the processing of body-related somatosensory signals (Naito et al., 2005).
Table 2. Differences between heavier and lighter than predicted (interaction term)

\begin{tabular}{|c|c|c|c|c|c|c|}
\hline \multirow[b]{2}{*}{ Anatomical region } & \multicolumn{3}{|c|}{$\begin{array}{l}\text { Talairach } \\
\text { coordinates }\end{array}$} & \multirow[b]{2}{*}{ Peak Zscore } & \multirow[b]{2}{*}{ Cluster size } & \multirow[b]{2}{*}{$p$ value $^{a}$} \\
\hline & $x$ & $y$ & $z$ & & & \\
\hline \multicolumn{7}{|c|}{ (Change to heavier - No change heavy) - (Change to lighter - No change light) } \\
\hline Left central sulcus (M1/S1) & -45 & -18 & 51 & $4.78^{b}$ & & \\
\hline Left precentral gyrus (M1) & -27 & -2 & 63 & $4.55^{b}$ & 222 & $<0.001$ \\
\hline Left postcentral gyrus (S1) & -24 & -3 & 66 & $4.10^{c}$ & & \\
\hline \multicolumn{7}{|c|}{$\begin{array}{l}\text { (Change to lighter }- \text { No change light) }- \text { (Change to heavier - No change heavy) } \\
\text { Right lateral cerebellum }\end{array}$} \\
\hline (lobule VI/Crus I) & 15 & $-7 \varepsilon$ & $3-2$ & 4.09 & 46 & 0.038 \\
\hline
\end{tabular}

${ }^{a} p$ values are given at the cluster level after correction for multiple comparisons in the entire brain space.

${ }^{b} p<0.01$ corrected based on small volume correction based on sphere around peak voxel taken from Schmitz et al. (2005).

'Secondary peak corresponding to $p<0.001$ uncorrected.

In our previous study (Schmitz et al., 2005), we also found activity in the right inferior frontal cortex (area 44) in lifting series with predictable or unpredictable weight changes. This finding could not be confirmed in the present study. A major difference between the two studies is that the previous experiment used a block design averaging data during a $40 \mathrm{~s}$ timeframe, whereas the event-related technique used in the present study identified the BOLD response evoked by a single lift. Although one should be cautious when interpreting negative findings in neuroimaging, this may indicate that the inferior frontal cortex has a more sustained pattern of activity across the lifting series. However, the difference may also simply reflect the fact that the event-related design is less sensitive (efficient) than the block design.

\section{Corrective responses and their neural correlates}

The right ipsilateral cerebellum was only active when the weight of the object unexpectedly changed to a light weight (Fig. $4 B$, Table 2). Thus, the cerebellar activity may reflect the brisk termination of the fingertip force increase, which is triggered by tactile afferents in the fingertips and occurs $\sim 100 \mathrm{~ms}$ after the premature liftoff (Westling and Johansson, 1987). The cerebellum has inhibitory outputs to several different cortical and subcortical regions, including thalamocortical projections to the primary motor cortex (Zemanick et al., 1991; Kelly and Strick, 2003), that could mediate the seen motor response. The thalamocortical projections to the motor cortex receive input from lobule VI of the cerebellum (Kelly and Strick, 2003), which is the same region where the peak of the activity was located in the present study (i.e., lobule VI at the border zone between the medial and lateral cerebellum; $x=+15)$. The cerebellum can also indirectly control both the lateral and the medial descending motor pathways via various nuclei in the brain stem. The finding that patients with cerebellar disorders use increased grip forces in various manipulative tasks (Nowak et al., 2002; Rost et al., 2005) is well in line with the interpretation of the present findings that the cerebellum exerts an inhibitory control on the force output during object manipulation.

An alternative interpretation is that the right cerebellar activity reflects the processing of unpredicted sensory input that triggers the brisk force termination, rather than the inhibitory motor response as such. When the object is lighter than expected, there is a premature lift of the object and the hand is quickly accelerated upward inducing a multitude of somatosensory signals of the rapidly moving limb (see overshoot in grip force and position signal in Fig. 2). In contrast, when the object is heavier than 
expected, the actual triggering is the absence of a predicted sensory signal reporting liftoff. The cerebellar response could reflect a different type of sensory feedback and error signal that triggers these corrective responses. It is unlikely that the cerebellar activity reflected differences in total afferent sensory input or total motor output, because no activity was observed when we compared lifting the heavy and the light objects (No change heavy - No change light; $p>0.001$ uncorrected). But regardless of whether the right cerebellar activation reflects the processing of the unpredicted sensory feedback, or the brisk termination of the force output that is triggered by this feedback, our results demonstrate that this region is involved in the corrective response when the object is lighter than predicted.

The stronger activity in the hand sections of left (contralateral) M1 and S1 during the lifts when the object was unpredictably heavy, and the initially programmed lift failed to lift the object, corresponds well with the triggered force correction increasing the fingertip forces. The correction is characterized by a slow and pulsatile increase until the weight of the object is overcome. It is important to point out that the total force increase during the dynamic part of the lift and the force output during the subsequent static phase when the object is held in the air (Figs. 1, 2 ) were similar in both the erroneous and the adequate programmed trials for the heavy weight. Thus, the M1 activity in question reflects the corrective force increase during the unpredictable weight change and not the total force output. Slow movements of the fingers are characterized by $8-10 \mathrm{~Hz}$ discontinuities (Vallbo and Wessberg, 1993; Wessberg and Vallbo, 1995) to which muscle spindles vigorously respond, and these discontinuities were also observed in this study (Fig. 1, grip force rate profile). In man, the S1 and M1 are activated by muscle spindle responses from the muscles of the contralateral hand (Naito et al., 1999, 2002), and in monkeys, neurons in both M1 (Lemon et al., 1976; Fetz et al., 1980; Soso and Fetz, 1980; Lemon, 1981) and S1 (Iwamura et al., 1983, 1993) respond to muscle stretch. Thus, the activation of these areas could reflect increased sensory feedback arising from the pulsatile force increase, as well as the generation of the corrective force responses.

\section{References}

Binkofski F, Buccino G, Posse S, Seitz RJ, Rizzolatti G, Freund H (1999) A fronto-parietal circuit for object manipulation in man: evidence from an fMRI study. Eur J Neurosci 11:3276-3286.

Blakemore SJ, Sirigu A (2003) Action prediction in the cerebellum and in the parietal lobe. Exp Brain Res 153:239-245.

Blakemore SJ, Frith CD, Wolpert DM (2001) The cerebellum is involved in predicting the sensory consequences of action. NeuroReport 12:1879-1884.

Cavada C, Goldman-Rakic PS (1989) Posterior parietal cortex in rhesus monkey: II. Evidence for segregated corticocortical networks linking sensory and limbic areas with the frontal lobe. J Comp Neurol 287:422-445.

Clower DM, West RA, Lynch JC, Strick PL (2001) The inferior parietal lobule is the target of output from the superior colliculus, hippocampus, and cerebellum. J Neurosci 21:6283-6291.

Dettmers C, Fink GR, Lemon RN, Stephan KM, Passingham RE, Silbersweig D, Holmes A, Ridding MC, Brooks DJ, Frackowiak RS (1995) Relation between cerebral activity and force in the motor areas of the human brain. J Neurophysiol 74:802-815.

Downar J, Crawley AP, Mikulis DH, Davis KD (2000) A multimodal cortical network for the detection of changes in the sensory enviroment. Nat Neurosci 3:277-283.

Doya K (1999) What are the computations of the cerebellum, the basal ganglia and the cerebral cortex? Neural Netw 12:961-974.

Ehrsson HH, Fagergren A, Jonsson T, Westling G, Johansson RS, Forssberg H (2000) Cortical activity in precision- versus power-grip tasks: an fMRI study. J Neurophysiol 83:528-536.
Ehrsson HH, Fagergren E, Forssberg H (2001) Differential fronto-parietal activation depending on force used in a precision grip task: an fMRI study. J Neurophysiol 85:2613-2623.

Ehrsson HH, Fagergren A, Johansson RS, Forssberg H (2003) Evidence for the involvement of the posterior parietal cortex in coordination of fingertip forces for grasp stability in manipulation. J Neurophysiol 90: $2978-2986$.

Fetz EE, Finocchio DV, Baker MA, Soso MJ (1980) Sensory and motor responses of precentral cortex cells during comparable passive and active joint movements. J Neurophysiol 43:1070-1089.

Ghosh S, Gattera R (1995) A comparison of the ipsilateral cortical projections to the dorsal and ventral subdivisions of the macaque premotor cortex. Somatosens Mot Res 12:359-378.

Godschalk M, Lemon RN, Kuypers HG, Ronday HK (1984) Cortical afferents and efferents of monkey postarcuate area: an anatomical and electrophysiological study. Exp Brain Res 56:410-424.

Gordon AM, Forssberg H, Johansson RS, Westling G (1991) The integration of haptically acquired size information in the programming of precision grip. Exp Brain Res 83:483-488.

Gordon AM, Westling G, Cole KJ, Johansson RS (1993) Memory representations underlying motor commands used during manipulation of common and novel objects. J Neurophysiol 69:1789-1796.

Iwamura Y, Tanaka M, Sakamoto M, Hikosaka O (1983) Functional subdivisions representing different finger regions in area 3 of the first somatosensory cortex of the conscious monkey. Exp Brain Res 51:315-326.

Iwamura Y, Tanaka M, Sakamoto M, Hikosaka O (1993) Rostrocaudal gradients in the neuronal receptive field complexity in the finger region of the alert monkey's postcentral gyrus. Exp Brain Res 92:360-368.

Jenmalm P, Johansson RS (1997) Visual and somatosensory information about object shape control manipulative fingertip forces. J Neurosci 17:4486-4499.

Johansson RS (1998) Sensory input and control of grip. In: Sensory guidance of movement, Novartis Foundation Symposium 218, pp 45-59. Chichester, UK: Wiley.

Johansson RS, Cole KJ (1992) Sensory-motor coordination during grasping and manipulative actions. Curr Opin Neurobiol 2:815-823.

Johansson RS, Westling G (1988) Coordinated isometric muscle commands adequately and erroneously programmed for the weight during lifting task with precision grip. Exp Brain Res 71:59-71.

Kawato M (1999) Internal models for motor control and trajectory planning. Curr Opin Neurobiol 9:718-727.

Kelly RM, Strick PL (2003) Cerebellar loops with motor cortex and prefrontal cortex of a nonhuman primate. J Neurosci 23:8432-8444.

Kuhtz-Buschbeck JP, Ehrsson HH, Forssberg H (2001) Human brain activity in the control of fine static precision grip forces: an fMRI study. Eur J Neurosci 14:382-390.

Lemon RN (1981) Functional properties of monkey motor cortex neurones receiving afferent input from the hand and fingers. J Physiol (Lond) 311:497-519.

Lemon RN, Hanby JA, Porter R (1976) Relationship between the activity of precentral neurones during active and passive movements in conscious monkeys. Proc R Soc Lond B Biol Sci 194:341-373.

Lemon RN, Baker SN, Davis JA, Kirkwood PA, Maier MA, Yang HS (1998) The importance of the cortico-motoneuronal system for control of grasp. Novartis Found Symp 218:202-215.

Maier MA, Bennett KM, Hepp-Reymond MC, Lemon RN (1993) Contribution of the monkey corticomotoneuronal system to the control of force in precision grip. J Neurophysiol 69:772-785.

Monzee J, Smith AM (2004) Responses of cerebellar interpositus neurons to predictable perturbations applied to an object held in a precision grip. J Neurophysiol 91:1230-1239.

Naito E, Ehrsson HH, Geyer S, Zilles K, Roland PE (1999) Illusory arm movements activate cortical motor areas: a positron emission tomography study. J Neurosci 19:6134-6144.

Naito E, Roland PE, Ehrsson HH (2002) I feel my hand moving: a new role of the primary motor cortex in somatic perception of limb movement. Neuron 36:979-988.

Naito E, Roland PE, Grefkes C, Choi HJ, Eickhoff S, Geyer S, Zilles K, Ehrsson $\mathrm{HH}$ (2005) Dominance of the right hemisphere and role of area 2 in human kinesthesia. J Neurophysiol 93:1020-1034.

Neal JW, Pearson RC, Powell TP (1990) The ipsilateral cortico-cortical con- 
nections of area 7b, PF, in the parietal and temporal lobes of the monkey. Brain Res 524:119-132.

Nowak DA, Hermsdörfer J, Marquardt C, Fuchs HH (2002) Grip and load force coupling during discrete vertical arm movements with a grasped object in cerebellar atrophy. Exp. Brain Res 145:28-39.

Picard N, Smith AM (1992) Primary motor cortical responses to perturbations of prehension in the monkey. J Neurophysiol 68:1882-1894.

Rost K, Nowak DA, Timmann D, Hermsdörfer J (2005) Preserved and impaired aspects of predictive grip force control in cerebellar patients. Clin Neurophysiol 116:1405-1414.

Schmitz C, Jenmalm P, Ehrsson HH, Forssberg H (2005) Brain activity during predictable and unpredictable weight changes when lifting objects. J Neurophysiol 93:1498-1509.

Soso MJ, Fetz EE (1980) Responses of identified cells in postcentral cortex of awake monkeys during comparable active and passive joint movements. J Neurophysiol 43:1090-1110.

Vallbo AB, Wessberg J (1993) Organization of motor output in slow finger movements in man. J Physiol (Lond) 469:673-691.

Wessberg J, Vallbo AB (1995) Coding of pulsatile motor output by human musele afferents during slow finger movements. J Physiol (Lond) 485:271-282.

Westling G, Johansson RS (1987) Responses in glabrous skin mechanoreceptors during precision grip in humans. Exp Brain Res 66:128-140.

Wolpert DM, Ghahramani Z (2000) Computational principles of movement neuroscience. Nat Neurosci [Suppl] 3:1212-1217.

Zemanick MC, Strick PL, Dix RD (1991) Direction of transneuronal transport of herpes simplex virus 1 in the primate motor system is straindependent. Proc Natl Acad Sci USA 88:8048-8051. 